\title{
A New Splicing Isoform of Cacna2d4 Mimicking the Effects of c.2451insC Mutation in the Retina: Novel Molecular and Electrophysiological Insights
}

\author{
Niccolò Bacchi, ${ }^{1}$ Andrea Messina, ${ }^{1}$ Verena Burtscher ${ }^{2}$ Erik Dassi, ${ }^{1}$ Giovanni Provenzano, ${ }^{1}$ \\ Yuri Bozzi, ${ }^{1,3}$ Gian Carlo Demontis, ${ }^{4}$ Alexandra Koschak, ${ }^{2,5}$ Michela A. Denti, ${ }^{1,6}$ \\ and Simona Casarosa ${ }^{1,3}$
}

${ }^{1}$ Centre for Integrative Biology (CIBIO), University of Trento, Trento, Italy

${ }^{2}$ Medical University of Vienna, Center for Physiology and Pharmacology, Department of Neurophysiology and Pharmacology, Vienna, Austria

${ }^{3}$ Neuroscience Institute-National Research Council (CNR), Pisa, Italy

${ }^{4}$ Department of Pharmacy, University of Pisa, Pisa, Italy

${ }^{5}$ University of Innsbruck, Institute of Pharmacy, Pharmacology and Toxicology, Center for Chemistry and Biomedicine, Innsbruck, Austria

${ }^{6}$ Neuroscience Institute-National Research Council (CNR), Padova, Italy

Correspondence: Simona Casarosa, University of Trento, Via Sommarive 9, 38123 Trento, Italy; simona.casarosa@unitn.it.

Michela Denti, University of Trento, Via Sommarive 9, 38123 Trento, Italy; michela.denti@unitn.it.

Alexandra Koschak, University of Innsbruck, Innrain80-82/III, 6020 Innsbruck, Austria; alexandra.koschak@uibk.ac.at.

Submitted: January 9, 2015

Accepted: May 22, 2015

Citation: Bacchi N, Messina A, Burtscher V, et al. A new splicing isoform of Cacna2d4 mimicking the effects of c.2451insC mutation in the retina: novel molecular and electrophysiological insights. Invest $O p h$ thalmol Vis Sci. 2015;56:4846-4856. DOI:10.1167/iovs.15-16410
Purpose. Mutations in CACNA2D4 exon 25 cause photoreceptor dysfunction in humans (c.2406C $\rightarrow$ A mutation) and mice (c.2451insC mutation). We investigated the feasibility of an exon-skipping therapeutic approach by evaluating the splicing patterns and functional role of targeted exons.

Methods. Splicing of the targeted $\alpha_{2} \delta_{4}(C A C N A 2 D 4)$ exons in presence and absence of the mutation was assessed by RT-PCR in vivo on mouse retinae and in vitro in HEK293T cells using splicing-reporter minigenes. Whole-cell patch-clamp recordings were performed to evaluate the impact of different Cacna2d4 variants on the biophysical properties of Cav1.4 Ltype calcium channels (CACNA1F).

REsults. Splicing analysis revealed the presence of a previously unknown splicing isoform of $\alpha_{2} \delta_{4}$ in the retina that truncates the gene open reading frame (ORF) in a similar way as the c.2451insC mutation. This isoform originates from alternative splicing of exon 25 (E25) with a new exon (E25b). Moreover, the c.2451insC mutation has an effect on splicing and increases the proportion of transcripts including E25b. Our electrophysiological analyses showed that only full-length $\alpha_{2} \delta_{4}$ was able to increase Cav1.4/ $\beta 3$-mediated currents while all other $\alpha_{2} \delta_{4}$ variants did not mediate such effect.

Conclusions. The designed exon-skipping strategy is not applicable because the resulting skipped $\alpha_{2} \delta_{4}$ are nonfunctional. $\alpha_{2} \delta_{4}$ E25b splicing variant is normally present in mouse retina and mimics the effect of c. 2451 ins C mutation. Since this variant does not promote significant Cav1.4-mediated calcium current, it could possibly mediate a different function, unrelated to modulation of calcium channel properties at the photoreceptor terminals.

Keywords: $\mathrm{Ca}^{2+}$ channels, exon-skipping, splicing isoforms, Cacna2d4
$\mathrm{E}^{\mathrm{x}}$ xon-skipping therapeutic approaches offer a unique opportunity for intervention in patients affected by retinal dystrophies, as different types of mutations can be potentially treated in a patient-specific way. ${ }^{1}$ Among the potential applications, it is possible to use different antisense molecular tools to induce intronization of selected exons. This effect can be achieved by masking cis-acting elements on the pre-mRNA important for an exon to be recognized as such by the splicing machinery. ${ }^{2}$ This strategy can be beneficial where the targeted exons are carrying nonsense mutations or insertions/deletions leading to frameshift. However, the precise splicing pattern of the mutated target exon(s) and the functionality of the skipped protein need to be carefully evaluated. This approach has been successfully applied to Duchenne muscular dystrophy (DMD). Dystrophin function, impaired in DMD by different types of mutations affecting the reading frame of the gene, can in fact be restored by skipping of different exons. This results in a shorter version of the protein, lacking the portion encoded by the skipped exons but maintaining the reading frame after the deletion. ${ }^{3}$ We selected the CACNA2D4 gene as a potential therapeutic target in the retina: This gene encodes for $\alpha_{2} \delta_{4}$, a member of $\alpha_{2} \delta$ accessory subunits of high voltage activated (HVA) calcium channels. ${ }^{4}$ High voltage activated calcium channels are multiprotein complexes composed of an $\alpha_{1}$ subunit, which constitutes the channel pore, and different accessory subunits $\left(\alpha_{2} \delta, \beta\right.$, and in some cases $\left.\gamma\right) \cdot \alpha_{2} \delta$ subunits are known to regulate HVA calcium channels mainly by increasing channel presence on the cell membrane, and by modulating channel gating properties. ${ }^{5}$ They are translated as a single protein but then cleaved into $\alpha_{2}$ and $\delta$ peptides, which 
A.

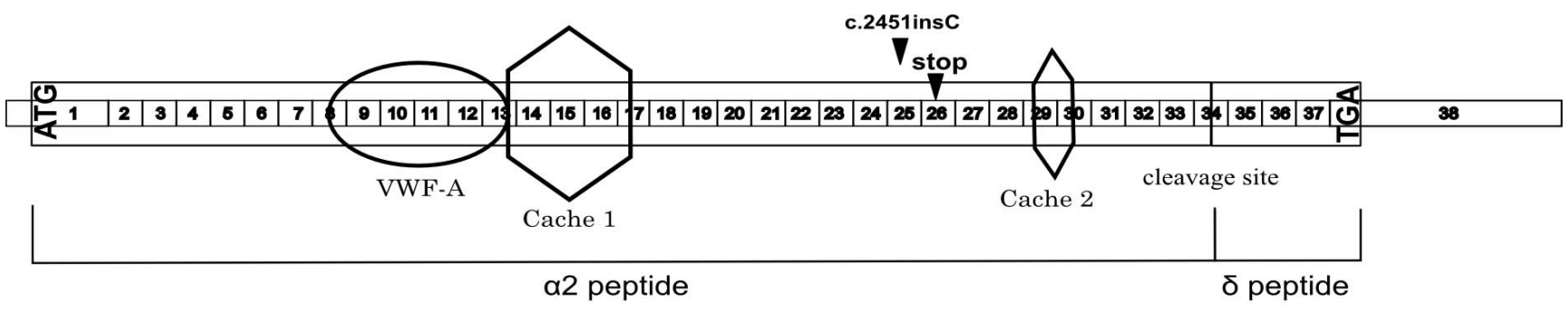

B.

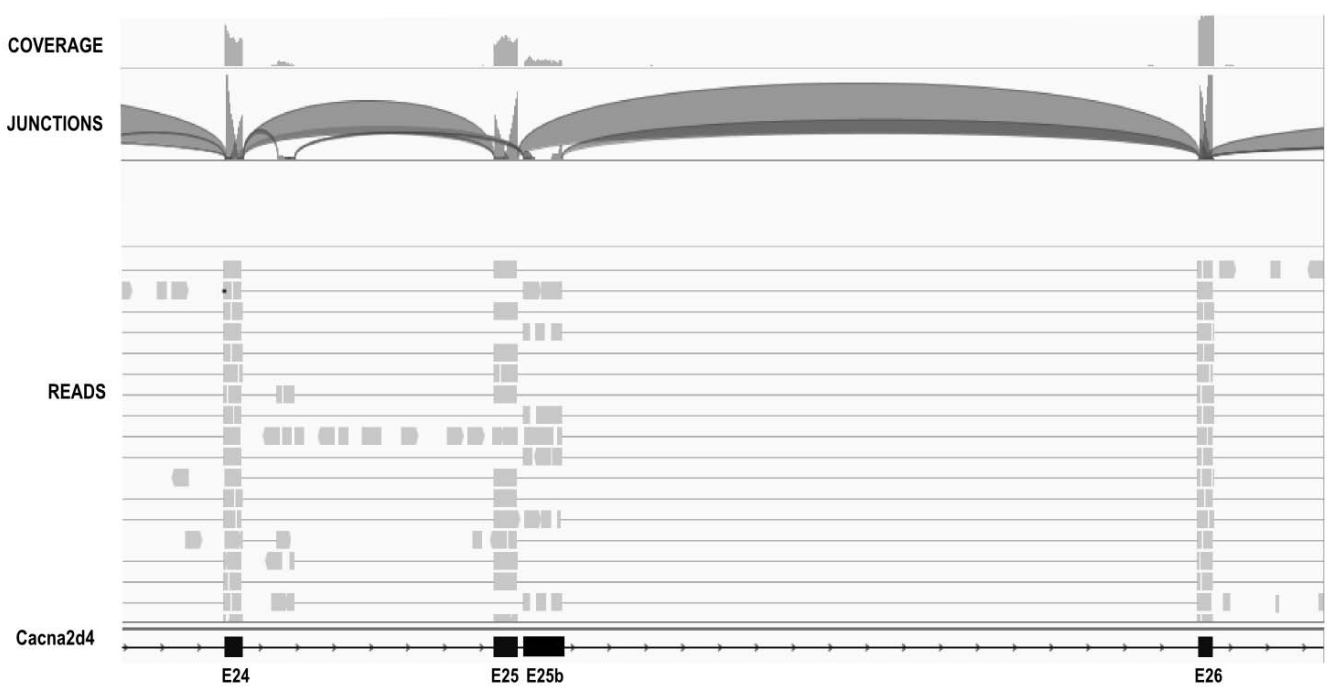

C.

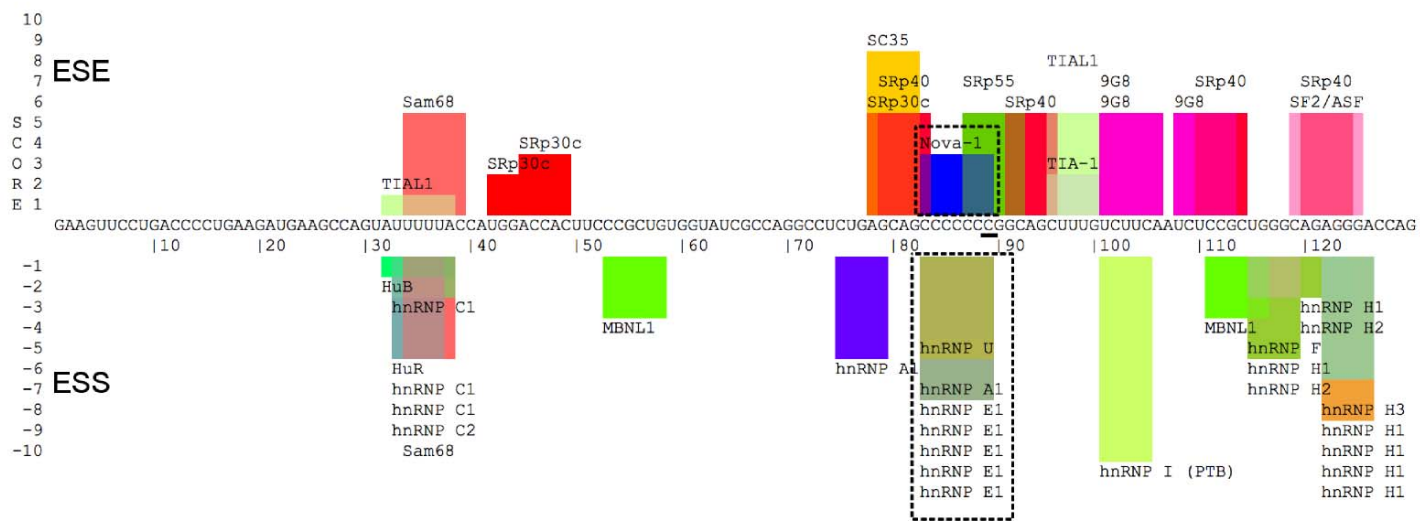

Figure 1. Scheme of Cacna2d4 transcript and computational analysis of exon 25 splicing. (A) Schematic representation of Cacna2d4 transcript (not in scale). The various exons are numbered. Positions of the von Willebrand factor A (VWF-A) domain ${ }^{43,44}$ and of the two Cache ${ }^{45}$ domains of the protein are shown. The murine c.2451insC mutation site and the resulting nonsense codon are indicated by black arrowheads. (B) IGV visualization of the RNA-seq eye sample GSM737548 aligned to Cacna2d4 genomic sequence. Position of E25b has been included. Junctions represent reads spanning over two exons: Alternative splicing of E25 and E25b can be observed. (C) SpliceAid prediction of E25 exonic splicing enhancers (ESE, positive scores) and silencers (ESS, negative scores). Predictions present only on the mutant E25 are boxed. The mutation mainly creates ESSs with high scores. The location of the insertion on E25 is underlined in black.

are subsequently joined together by a disulphide bond. The $\alpha_{2}$ peptide is extracellular, while the $\delta$ mediates membrane anchoring. ${ }^{6}$ In the retina, $\alpha_{2} \delta_{4}$ is the main accessory subunit ${ }^{7-9}$ where it is suggested to form a complex with Cav1.4 $\alpha_{1}$ and $\beta_{2}$ subunits, ${ }^{10}$ since mutations in all these genes are linked to retinal dystrophies. ${ }^{7,9,11,12}$ So far, two mutations in Cacna2d4 exon 25 have been reported to cause cone and cone-rod dystrophies in a human family and in a spontaneous mouse model. ${ }^{7,8}$ Both mutations are expected to result in a truncated protein, likely to be nonfunctional due to the loss of the $\delta$ peptide and other functional domains downstream of the mutation (Fig. 1A). Since the skipping of the sole mutationcarrying exon 25 in $\alpha_{2} \delta_{4}$ would result in frameshift, skipping of exons 23 to $26\left(\alpha_{2} \delta_{4} \Delta \mathrm{E} 23-26\right)$ or of exons 23 to $25\left(\alpha_{2} \delta_{4} \Delta \mathrm{E} 23\right.$ $25)$ is needed to restore the reading frame downstream of the skipped exons. We hypothesized that this strategy would result 
in a rescued protein missing the skipped exons, but retaining the $\delta$ peptide and other structural elements. Moreover, the targeted exons do not contain any known domain important for $\alpha_{2} \delta$ function. The presence of a mouse model ${ }^{7}$ and the ability to test the functionality of the skipped protein by electrophysiology offer a clear advantage in assessing the feasibility of this exon-skipping approach. We thus generated constructs lacking these exons and tested their functionality by electrophysiology in cells in which the whole channel complex had been reconstituted.

\section{MeTHODS}

\section{Computational Analysis of Cacna2d4 E25 Splicing}

Mouse eye RNA-sequencing samples were downloaded from the Gene Expression Omnibus ${ }^{13}$ (dataset ID GSE38359: sample IDs GSM945628, GSM945631, and GSM945634; dataset ID GSE29752: samples GSM737548 and GSM737550). Reads were trimmed at the $3^{\prime}$ end by removing nucleotides having Q score $<30$ with Trimmomatic, ${ }^{14}$ and then mapped to the mouse genome (mm10 assembly) with TopHat $2 .{ }^{15}$ The resulting alignment and the observed exon-exon junctions were eventually visualized with Integrative Genomics Viewer (IGV). ${ }^{16}$

The genomic sequence of Cacna2d4 between E22 and E27 was analyzed using NNSPLICE $0.9,{ }^{17}$ and scores for the different splice sites were reported.

Cacna2d4 and CACNA2D4 E25 sequences were analyzed using SpliceAid ${ }^{18}$ in presence and absence of the c.2451insC mutation, obtaining predictions of differentially binding splicing regulatory elements.

\section{Animals}

Cacna2d 4 mutant and wild-type (WT) mice with C57BL/10 background $^{7}$ (2 months old) were used for retinal tissue extraction. C57BL/6 mice (3-5 months old) were used for analysis of Cacna2d4 $\Delta \mathrm{E} 16$ expression in the central nervous system (CNS). Experiments were approved by the Italian Ministry of Health and conducted in conformity with the European Community Directive 2010/63/EU and the ARVO Statement for the Use of Animals in Ophthalmic and Vision Research.

\section{Cloning}

Cacna2d4 $\Delta \mathrm{E} 16$ clone (GenBank: BC141091.1; IMAGE ID 9055703) was ordered from Source Bioscience (Nottingham, UK), amplified by PCR using primers For 5'-GACTGC TAGCCACTTGCATGC and Rev 5'-CTTGTCGACGTCAGATGG GAT, and cloned into pIRES2-EGFP (Clontech, Mountain View, CA, USA) using NheI and SalI restriction sites. Exon 16 was subsequently amplified from murine genomic DNA using primers For 5'-AGCTGGCACCCCGATATAAGCTTGGGGTG CATGGC and Rev 5'-AGCTTCTTGCCTTCTCTGTA CAAAGGTCGGAGGTCAG. The resulting PCR fragment was used as megaprimer ${ }^{19}$ for site-directed mutagenesis (Quickchange II site-directed mutagenesis kit; Agilent, Santa Clara, CA, USA) and added to Cacna2d4 $\Delta \mathrm{E} 16$ to generate Cacna2d 4 fulllength $\left(\alpha_{2} \delta_{4}\right)$. The c.2451insC mutation on E25 was introduced by site-directed mutagenesis of Cacna2d4 using primers For 5'GAGCAGCCCCCCCGGCAGCTTTGTCTTC and Rev 5'-GAAGA CAAAGCTGCCGGGGGGGCTGCTC. The obtained construct was named $\alpha_{2} \delta_{4}$ MUT. E25b was amplified from mouse retina cDNA using primers For 5'-AGAGTCAGAGCCTGGCGTGG and Rev 5'-CTGCTGCAATGGCCGTCTTCC. The PCR product was extracted from gel to avoid E25-containing transcript and purified. The product was then used as megaprimer for sitedirected mutagenesis to obtain E25b inclusion in place of E25 $\left(\alpha_{2} \delta_{4}\right.$ E25b). The $\alpha_{2} \delta_{4}$ full-length construct has been subsequently utilized as template for the generation of $\alpha_{2} \delta_{4} \Delta \mathrm{E} 23-25$. For this intent, a PCR using primers For 5 '-atagcccaggcaagccagt and Rev 5'-actccaggtctggatccac was performed, followed by ligation of the PCR product. The $\alpha_{2} \delta_{4} \Delta \mathrm{E} 23-26 \mathrm{cDNA}$ construct was obtained from overlapping PCR on Cacna $2 d 4$ template. For this purpose two different primer pairs were used to originate the two overlapping fragments: For 5'-CTACGTGACTGCTAGC CACTTGCATGCCCAGGA and Rev 5'-ATCTGGATGCCCACA GACTCCAGGTCTGGATCC for first reaction; For 5'-GATCCA GACCTGGAGTCTGTGGGCATCCAGATG and Rev 5'-CTAC GACTCTTGGATCCGTCAGATGGGATGGAGTC for the second reaction. The two generated fragments were then purified and used together with the primer pair For 5'-GACTGCTAGC CACTTGCATGC and Rev 5'-CTTGTCGACGTCAGATGGGAT in a different PCR reaction to generate $\alpha_{2} \delta_{4} \Delta \mathrm{E} 23-26$. The Cacna2d4 minigene construct was assembled from four fragments amplified from mouse genomic DNA and digested with specific restriction enzymes. For a detailed list of utilized primers and restriction enzymes see Supplementary Table S1. The resulting digested and purified fragments were ligated together with a pCDNA3-Luc2 vector digested with HindIII and XboI. The introduction of c.2451insC mutation into the minigene was performed by site-directed mutagenesis using the same primer pair utilized for $\alpha_{2} \delta_{4}$ MUT.

The CACNA2D4 human clone (GenBank: EU832243.1; IMAGE ID: 100067272) was ordered from Source Bioscience, amplified by PCR using primers For 5'- gaaccgtcagatccgctagcGTA CAAAAAAGCAGGCTCCACCATG and Rev 5' - gggcccgcggtaccgtc gactACCGCAGGAGTTGGGGCA, and cloned by Gibson assembly (New England Biotech, Ipswich, MA, USA) into a pIRES2-EGFP (Clontech) digested with NheI and Sall restriction enzymes. The generated construct was used as positive control in Western blots. All obtained clones were checked by sequencing.

\section{RNA Extraction and cDNA Synthesis}

Each tissue was dissected from three adult mice and pooled. Total RNA was extracted using Trizol Reagent (Life Technologies, Carlsbad, CA, USA), treated with DNase (Roche, Basel, Switzerland), and purified using NucleoSpin RNA columns (Macherey-Nagel, Düren, Germany). Complementary DNA synthesis was performed using random excimers (SuperScript VILO cDNA Synthesis Kit; Life Technologies). RNA extraction of transfected HEK293T cells was performed 2 days after transfection as described above.

\section{Cell Culture and Transfection Conditions}

Cells used for electrophysiological recordings were tsA-201, also called HEK293T. ${ }^{20,21}$ These are HEK293 cells that constitutively express the SV40 large T antigen to achieve higher level of expression from transfected plasmids. We kept, however, the distinct nomenclature used by the laboratories of origin since in electrophysiological studies tsA-201 is still the prevalent name adopted. HEK293T cells were maintained in Dulbecco's Modified Eagle Medium (DMEM) supplemented with $10 \%$ FBS, $2 \mathrm{mM}$ glutamine and $100 \mathrm{U} / \mu \mathrm{L}$ Pen/Strep and grown at $37^{\circ} \mathrm{C}, 10 \% \mathrm{CO}_{2}$. For transfection with minigenes, Lipofectamine 2000 (Life Technologies) was used. For electrophysiological recordings, tsA-201 cells were split into $6-\mathrm{cm}$ dishes 1 day before transfections. Transfection was performed using Turbofect transfection reagent (Thermo Scientific, Waltham, MA, USA) with $0.5 \mu \mathrm{g}$ Cav1.4 $\alpha_{1},{ }^{22} 0.5 \mu \mathrm{g} \beta_{3},{ }^{23}$ and $0.5 \mu \mathrm{g}$ different $\alpha_{2} \delta$ constructs in pIRES2-EGFP-expressing vectors and $1 \mu \mathrm{g}$ pUC. The $\alpha_{2} \delta_{1}$ sequence utilized was 
described previously. ${ }^{24}$ For $\alpha_{2} \delta_{1}{ }^{24}$ transfections, $0.05 \mu \mathrm{g}$ of a Green fluorescent protein (GFP)-expressing plasmid was cotransfected. One day after transfection, cells were seeded at low confluence on $3-\mathrm{cm}$ dishes coated with poly-D-lysine and stored overnight at $30^{\circ} \mathrm{C}, 5 \% \mathrm{CO}_{2}$. Recordings were performed the following day. In experiments in which different $\alpha_{2} \delta$ constructs were present in the same transfection, the following amounts were used: $0.5 \mu \mathrm{g}$ Cav1.4 $\alpha_{1},{ }^{22} 0.5 \mu \mathrm{g} \beta_{3},{ }^{23}$ $0.5 \mu \mathrm{g}$ each different $\alpha_{2} \delta$ subunit, and $0.5 \mu \mathrm{g}$ pUC.

\section{RT-PCR and Densitometric Analysis}

The PCR protocol consisted of denaturation $\left(94^{\circ} \mathrm{C} ; 1\right.$ minute), 35 amplification cycles $\left(94^{\circ} \mathrm{C}, 30\right.$ seconds; $58^{\circ} \mathrm{C}, 30$ seconds; $72^{\circ} \mathrm{C}, 1$ minute), and final extension $\left(72^{\circ} \mathrm{C}, 7\right.$ minutes $)$; primers are reported in Supplementary Table S2. Densitometric analysis was conducted using Imagelab 2.0 software (Bio-Rad, Hercules, CA, USA). Band intensities, corrected for background, were normalized to the corresponding glyceraldehyde 3-phosphate dehydrogenase (Gapdh) values.

\section{RT-Quantitative PCR}

Reverse transcription-quantitative (q)PCR was performed in a C1000 thermal cycler with a CFX384 real-time detection system (Bio-Rad), using the KAPA SYBR FAST master mix (Kapa Biosystems, Cape Town, South Africa). Gapdb was used as a reference gene for normalization, using the $\Delta \mathrm{Ct}$ method. Primers are reported in Supplementary Table S2. Data were analyzed using the CFX Manager 2.1 software (Bio-Rad). For direct comparison of E25 and E25b transcript levels, amplification efficiency and $y$-intercept with the two primer pairs were compared using serial dilutions of plasmid containing E25 or E25b. Equivalence of the obtained slopes and $y$ intercept was assessed with extra sum-of-square $F$ test. Data are shown as $2^{\wedge}(-\Delta \mathrm{Ct}(\mathrm{E} 25)) / 2^{\wedge}(-\Delta \mathrm{Ct}(\mathrm{E} 25 \mathrm{~b}))$.

\section{Protein Extraction and Western Blot}

The tsA-201 cells transfected with all the constructs used for electrophysiological analyses as described above were detached from 6-cm dishes using PBS, centrifuged briefly to remove PBS, and lysed with $100 \mu \mathrm{L}$ lysis buffer $(50 \mathrm{mM}$ Tris- $\mathrm{HCl}$ pH 8, $150 \mathrm{mM} \mathrm{NaCl}$, Tergytol-type NP-40 (NP-40) 1\%, Nadeoxycholate $0.25 \%, 1 \mathrm{mM}$ EDTA, $2 \mathrm{mM}$ Phenylmethanesulfonyl fluoride (PMSF), $2 \mathrm{mM}$ sodium orthovanadate, $0.1 \mathrm{M}$ protease inhibitors cocktail [Sigma-Aldrich Corp., St. Louis, MO, USA]). Protein concentration was assessed using Pierce BCA protein assay kit (Thermo Scientific). One hundred micrograms proteins $(50 \mu \mathrm{g}$ in the case of transfections with human $\alpha_{2} \delta_{4}$ ) was run on a precast $3 \%$ to $8 \%$ Tris-Acetate gel (Life Technologies). Proteins were transferred to a Hybond ECL nitrocellulose membrane (Sigma-Aldrich Corp.) using a transfer buffer (25 mM Tris-HCl, $192 \mathrm{mM}$ glycine, 20\% methanol, $0.1 \%$ SDS). The membrane was blocked for 1 hour at room temperature by exposure to $5 \%$ nonfat dry milk in $20 \mathrm{mM}$ Tris-HCl pH 8, 0.5 M NaCl, 0.1\% Tween-20 (Sigma-Aldrich Corp.). The membrane was then incubated overnight at $4^{\circ} \mathrm{C}$ with rabbit polyclonal anti-human CACNA2D4 antibody, 1:400 (Sigma-Aldrich Corp.) or for 1 hour at room temperature with mouse monoclonal anti-rat $\beta$-tubulin antibody, 1:10,000 (Santa Cruz, Dallas, TX, USA). As secondary antibody, a goat antirabbit horseradish peroxidase (HRP) conjugated IgG-HRP or a goat anti-mouse IgG-HRP, 1:10000 (Santa Cruz) was used for 1 hour at room temperature. A chemiluminescence-based kit (Amersham ECL Select Western Blotting Detection Reagent; GE Healthcare, Little Chalfont, UK) was used for detection.

\section{Electrophysiological Recordings}

Whole-cell patch-clamp experiments were performed using an Axopatch 700B amplifier (Molecular Devices, Sunnyvale, CA, USA). Borosilicate glass pipettes (external diameter $1.5 \mathrm{~mm}$, wall thickness $0.32 \mathrm{~mm}$; Science Products, Frankfurt/Main, Germany) were pulled using a micropipette puller (P97; Sutter Instruments, Novato, CA, USA) to obtain electrodes with a resistance of 1.5 to $3 \mathrm{M} \Omega$. pClamp10.2 (Molecular Devices) and Prism 5 (GraphPad, La Jolla, CA, USA) software was used for data analysis. Recordings were executed at $\mathrm{pH} 7.4$ (with $\mathrm{CsOH}$ ). The internal solution contained (in $\mathrm{mM}$ ) $135 \mathrm{CsCl}, 10$ HEPES, 10 EGTA, $1 \mathrm{MgCl}_{2}$, and the bath solution $15 \mathrm{CaCl}_{2}$ or $\mathrm{BaCl}_{2}, 10 \mathrm{HEPES}, 150$ choline-Cl, $1 \mathrm{MgCl}_{2}$. Recordings were performed at room temperature $\left(19^{\circ}-22^{\circ} \mathrm{C}\right)$. All voltages were corrected for a liquid junction potential of $-8 \mathrm{mV}$. In all protocols used, a holding potential of $-98 \mathrm{mV}$ was applied. To determine current densities, peak current amplitudes were normalized to the membrane capacitance. For each analyzed cell, the maximum observed current density $\left(\mathrm{CD}_{\max }\right)$ was reported. Protocols for the voltage dependence of activation and inactivation, as well as calcium-dependent inactivation, were used as described by Burtscher and colleagues. ${ }^{11}$

\section{Statistics}

Statistical analysis was performed with Prism 5 (GraphPad) and significance set at $P<0.05$ for all tests. The PCR data are represented as mean \pm SD and compared using unpaired twotailed Student's $t$-test. Standard deviation was calculated from biological replicates. Electrophysiological data are represented as mean \pm SEM and analyzed by Kruskal-Wallis test followed by Dunn's post hoc test for multiple comparisons. Mann-Whitney $U$ test was used for single comparisons.

\section{Results}

\section{Computational Analysis of Cacna2d4 Exon 25 Splicing}

We first investigated the feasibility of an exon-skipping approach for the c.2451insC mutation (previously annotated as c.2367insC) in exon 25 of Cacna2 $4^{7}$ (registered sequence NM_001033382.2). We first analyzed the splicing pattern of this and surrounding exons by an in silico approach using RNAseq data from four eyes and one retina of $\mathrm{C} 57 \mathrm{BL} / 6$ mice. Datasets showed that the canonical exon 25 (128 bp) was not always included in the final transcript. In these cases it was substituted by a new exon included between exon 24 and 26 (Fig. 1B). This putative exon of $205 \mathrm{bp}$, named E25b, is located in intron 25 in close proximity to exon 25 . If E25b is included instead of E25, it terminates the open reading frame (ORF) of Cacna2d4 in a way similar to the known mutation c.2451insC, as it carries a stop codon. E25b was present in fewer reads compared to E25, suggesting that it may be included in a smaller fraction of Cacna2d4 transcripts. To further corroborate the existence of this new exon we undertook a bioinformatics analysis of the $5^{\prime}$ and $3^{\prime}$ splice sites of E25b (Table 1) and discovered that the putative E25b showed higher prediction scores with respect to the canonical E25. In particular, the $3^{\prime}$ splice site of E25 is very weak, while the $3^{\prime}$ splice site of E25b is relatively strong. This strengthens the idea that E25b may be included in place of E25, thereby generating an alternatively spliced variant of Cacna2d4. Moreover, it suggests that nucleotide variations in E25 sequence may significantly affect its inclusion levels in mature transcripts, favoring E25b-containing transcripts. We hypothesized that the c.2451insC insertion could have such an effect, and compu- 
TABLE 1. Splice-Site Sequences and Scores for Cacna2d4 Exons 22 to 27

\begin{tabular}{|c|c|c|}
\hline & Score & Sequence $5^{\prime}>3^{\prime}$ \\
\hline E22 5'splice site & 0.52 & ctggagtgtgagttc \\
\hline E23 3'splice site & 0.92 & ttcctgtgactttaccctcaggtgacgaggaactggtgcgg \\
\hline E23 5'splice site & 0.85 & tctctgagtacgtac \\
\hline E24 3'splice site & 0.93 & tgttgttgctttgcctcgcagagagtcagagcctggcgtgg \\
\hline E24 5'splice site & 0.99 & ctgacaggtgagc c c \\
\hline E25 3'splice site & 0.43 & acatcaatcctactctcacaggaagttcctgacccctgaag \\
\hline E25 5'splice site & 0.92 & ggaccaggtaacgga \\
\hline E25b 3'splice site & 0.93 & cctctctgtgctcttccccagagtggaaaaagggagatgtg \\
\hline E25b 5'splice site & 0.99 & tcccaaggtgagatc \\
\hline E26 3'splice site & 0.98 & caataattctcatttttccagatagc coaggcaagc cagtg \\
\hline E26 5'splice site & 1.00 & gcagcaggtaagagc \\
\hline E27 3'splice site & 0.98 & cctccttcctgtttcctgcagctgtgggcatccagatgcaa \\
\hline
\end{tabular}

A perfect match with the canonical $3^{\prime}$ or $5^{\prime}$ splice site sequence is indicated by 1 . E25b scores are higher than those of E25, reflecting stronger splice sites.

tationally tested whether it might increase E25b inclusion using the SpliceAid web server. ${ }^{18}$ The output of the comparisons between WT and mutant E25 revealed how the insertion in E25 mainly establishes new exonic splicing silencers (ESS) (Fig. 1C). In fact, the prediction showed seven new ESS with scores between -4 and -7 in the mutant and only one new exonic splicing enhancer (ESE) with a score of 4 (Supplementary Table S3), all absent in the WT sequence. We thus predicted a change in the ratio of E25- and E25bcontaining transcripts between WT and mutant alleles, with the c.2451 insC insertion favoring E25b inclusion. These results were supported by analyzing the effect of human c.2406C $\rightarrow \mathrm{A}$ mutation in E25, where a predicted ESE is abolished and substituted with an ESS (Supplementary Fig. S1).

\section{In Vivo and In Vitro Characterization of Exon 25 Splicing}

We then verified the above hypothesis by analyzing E25 splicing in retinae of Cacna2d4 WT and mutant mice. ${ }^{7}$ We performed an RT-PCR using primers on exon 24 and 26 to confirm the presence of E25b along with E25 thanks to a clear difference in size (Fig. 2A). Densitometric analysis of PCR products revealed that $\mathrm{E} 25 \mathrm{~b}$ is less abundant than E25 in mature transcripts found in WT retinae. A significant decrease in E25-containing transcripts was instead observed in the mutant retina (Fig. 2B), while E25b levels remained constant. Reverse transcription-qPCR performed with specific primers for exon 25 and exon 25b confirmed that E25-containing transcripts were significantly diminished in mutant mice compared to WT; E25b-containing mRNAs were also reduced, although to a much lower extent (Fig. 2C). Quantification of E25- and E25b-containing transcripts revealed that the ratio between the two isoforms changed in favor of E25b in mutant animals (Fig. 2D).

We next assessed the effect of the mutation using a minigene reporter system, generated by cloning the exons surrounding E25 (exons 22-27) together with portions of intronic sequences (introns 22-26) in an expression cassette (Fig. 2E). Following minigene transfection in HEK293T cells, proper splicing between the different exons was observed by RT-PCR using primers on E22 and on the plasmid downstream of E27 (Fig. 2F). Minigene analysis also showed alternative splicing between E25 and E25b, as previously described in vivo. Interestingly, in HEK293T cells the effect of the mutation was to increase E25b inclusion without affecting E25 levels, as observed by densitometric analysis of the RT-PCR (Fig. 2G) and confirmed by RT-qPCR (Fig. $2 \mathrm{H}$ ) for the two isoforms. The ratio of E25 over E25b levels revealed that the ratio between the two isoforms tended to change in favor of E25b in mutant animals (Fig. 2I).

\section{Analysis of $\alpha_{2} \delta_{4} \Delta \mathrm{E} 16$ Splicing Isoform Expression in the CNS and Neuroendocrine System}

By database analyses, we found the presence of another putative splicing isoform of Cacna2d4, obtained from the sequencing of a whole-brain cDNA library. This isoform is characterized by the absence of exon $16\left(\alpha_{2} \delta_{4} \Delta \mathrm{E} 16\right.$; GenBank: BC141091.1), an exon that encodes for a portion of the first Cache domain of $\alpha_{2} \delta_{4}$, which is conserved in all known $\alpha_{2} \delta$ subunits. ${ }^{6}$ We thus investigated the presence of this isoform in retina, CNS, and the neuroendocrine system by RT-PCR (Fig. $3 \mathrm{~A})$. The $\Delta \mathrm{E} 16$ isoform was absent in the retina and in the pituitary gland, where all Cacna2d4 transcripts contained E16. Instead, $\triangle \mathrm{E} 16 \mathrm{mRNA}$ was the predominant isoform expressed in cerebellum, hippocampus, occipital cortex, and somatosensory cortex; both variants were present in hypothalamus. Densitometric analysis showed that Cacna2d4 is highly expressed in the retina, while lower levels were detected in other areas of the CNS (Fig. 3B).

\section{Functional Characterization of Different $\alpha_{2} \delta_{4}$ Variants}

Since the alteration of the $\alpha_{2} \delta_{4}$ ORF due to protein truncation (for $\alpha_{2} \delta_{4}$ MUT and $\alpha_{2} \delta_{4}$ E25b) or to partial deletion (for $\alpha_{2} \delta_{4}$ $\Delta \mathrm{E} 23-25, \alpha_{2} \delta_{4} \Delta \mathrm{E} 23-26$, and $\alpha_{2} \delta_{4} \alpha_{2} \delta_{4} \Delta \mathrm{E} 16$ ) could potentially result in protein instability, we confirmed by Western blot the presence of the different $\alpha_{2} \delta_{4}$ variants in tsA-201 cells after transfection. As shown in Figure $4, \alpha_{2} \delta_{4}$ is present in all conditions tested (lines 2-7) and absent in tsA-201 (line 1). The $\alpha_{2} \delta_{4}$ MUT and $\alpha_{2} \delta_{4}$ E25b show the smallest dimension, since in both cases the protein is truncated after exon 25. The $\alpha_{2} \delta_{4}$ $\Delta \mathrm{E} 16$ is instead very close to the WT, as it is missing only exon 16. The two constructs $\alpha_{2} \delta_{4} \Delta \mathrm{E} 23$ to 25 and $\alpha_{2} \delta_{4} \Delta \mathrm{E} 23$ to 26 show an intermediate size, since they lack three or four exons compared to the WT, but retain the portion of the protein downstream, which is instead not present in $\alpha_{2} \delta_{4}$ MUT and $\alpha_{2} \delta_{4}$ E25b.

The $\alpha_{2} \delta$ accessory subunits have different effects on HVA calcium channels: They primarily increase calcium influx by increasing the number of channels on the plasma membrane ${ }^{25-28}$; in addition, they fasten the inactivation kinetic of some HVA calcium channels. ${ }^{27,29,30}$ To identify how $\alpha_{2} \delta_{4}$ subunit modulates Cav1.4 L-type calcium currents, we 
A.

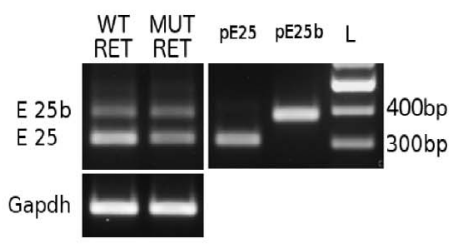

B.

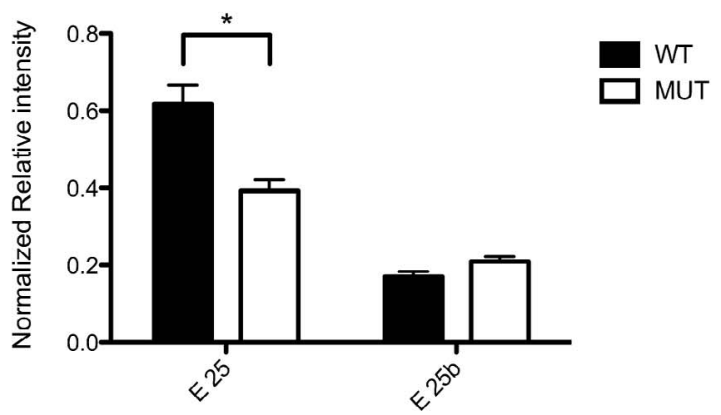

C.

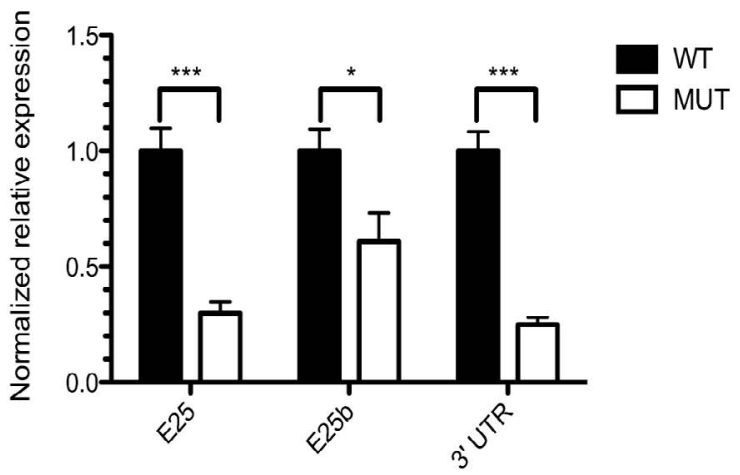

D.

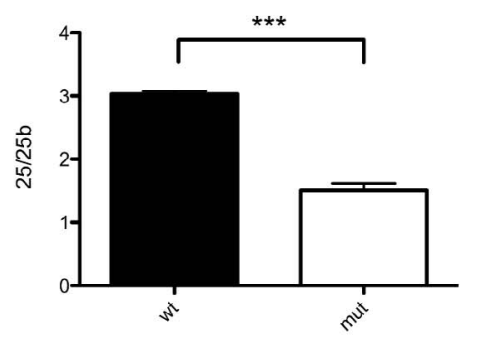

F.

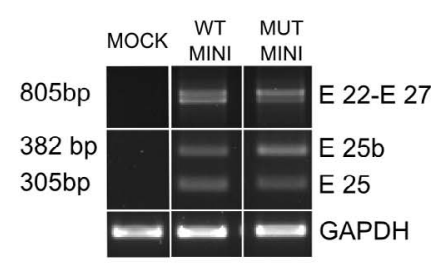

G.

H.
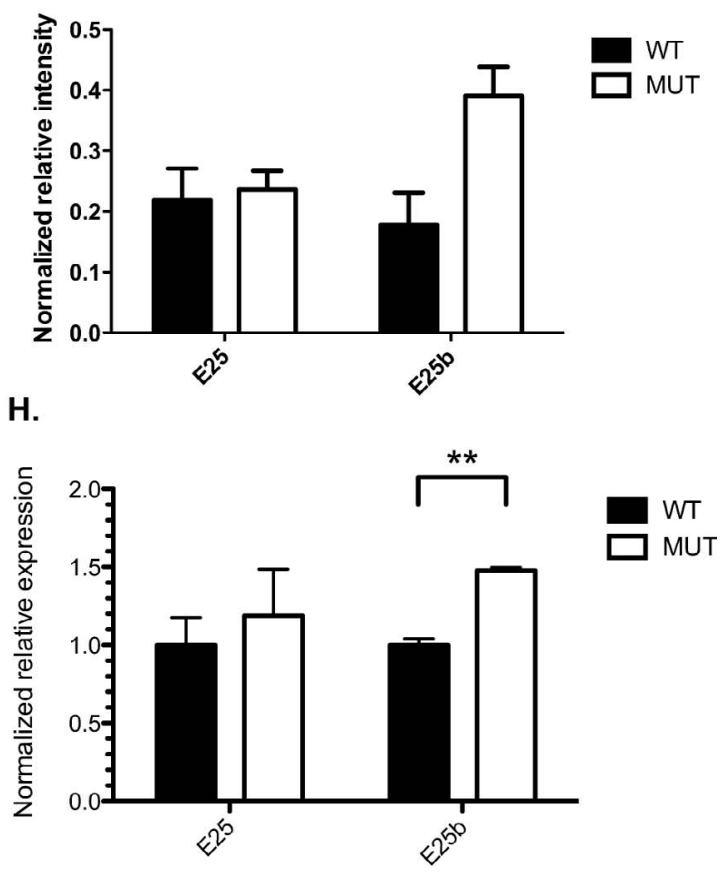

I.

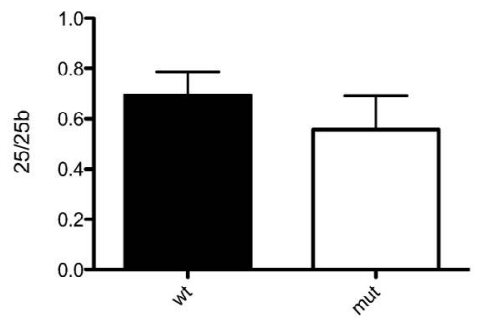

E.

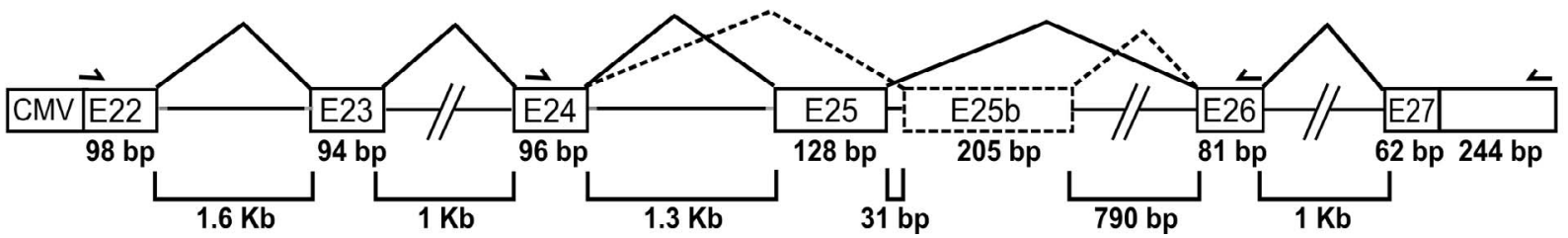

Figure 2. Qualitative and quantitative analysis of exon 25 alternative splicing in presence and absence of c.2451insC mutation. (A) Representative RT-PCR of WT and mutant mouse retinae. Plasmids containing Cacna2d4 sequence with E25 (pE25) or with E25b (pE25b) were used as controls. Presence of both E25 and E25b can be observed. (B) Densitometric analysis of the RT-PCR on WT and mutant mouse retinae reveals a reduction of E25-containing plasmid $(P<0.05)$. (C) Quantification of Cacna2d4 E25, E25b, and 3' UTR using RT-qPCR of WT and mutant mouse retinae $(n=3)$. A more pronounced reduction of E25 $(P<0.001)$ - compared to E25b $(P<0.05)$-containing transcripts was observed. (D) Relative abundance of E25- over E25b-containing transcripts in mouse retinae. The mutation favors E25b presence $(P<0.001)$. (E) Schematic representation of Cacna2d4 splicing-reporter minigene. The length of the different exons (boxes) and introns (straight lines) is shown. Alternative splicing events leading to E25b inclusion are reported with dashed lines. The position of primers used in RT-PCR is shown with arrows. (F) Representative RT-PCR of HEK293T cells transfected with WT (WT MINI) and mutant (MUT MINI) minigenes. The first line shows the occurrence of proper splicing between all exons included in the minigene. Two bands, close to $850 \mathrm{bp}$, are distinguishable. The lower is caused by E25 inclusion, the upper by E25b. The second line confirms the presence of E25b-containing transcripts alongside E25-containing ones. (G) Densitometric analysis of the RT-PCR on WT and mutant minigenes. The mutation acts on minigene splicing by favoring E25b inclusion. (H) Quantification of the two splicing isoforms (E25/ E25b) in presence or absence of the mutation, by means of RT-qPCR in cells transfected with the minigenes $(n=2)$. Data confirm that the mutation causes an increase in the levels of the splicing isoform including E25b $(P<0.01)$. (I) Relative abundance of E25- over E25b-containing transcripts in HEK293T cells transfected with minigene splicing reporter systems. The mutation favors E25b presence. 
A.

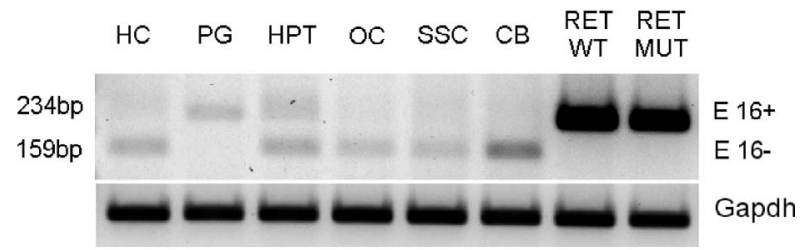

B.

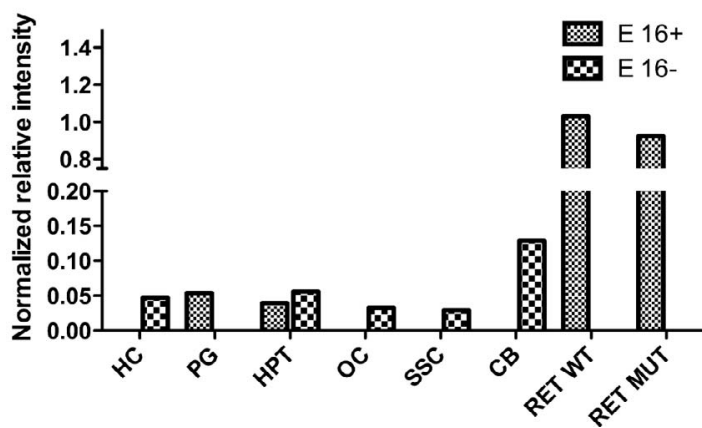

FIgURE 3. Analysis of $\alpha_{2} \delta_{4} \Delta \mathrm{E} 16$ splicing isoform presence in the CNS and neuroendocrine system. (A) RT-PCR with primers flanking E16 on mouse hippocampus (HC), pituitary gland (PG), hypothalamus (HPT), occipital cortex (OC), somatosensory cortex (SSC), cerebellum (CB), and WT (RET WT) and mutant (RET MUT) retina. (B) Densitometric analysis of RT-PCR results from the displayed image.

undertook whole-cell patch-clamp recordings in tsA-201 cells transfected together with $\beta_{3}$ subunits and compared its biophysical properties with the more frequently investigated $\alpha_{2} \delta_{1} \cdot{ }^{11,31}$ Coexpression of full-length $\alpha_{2} \delta_{4}$ resulted in a 3-fold increase in current density, similarly to $\alpha_{2} \delta_{1}$ (Figs. 5A, 5B, 5D). In addition, $\alpha_{2} \delta_{4}$ and $\alpha_{2} \delta_{1}$ displayed comparable voltagedependent activation and inactivation properties (Fig. $5 \mathrm{C}$; Table 2). We finally tested the effect of $\alpha_{2} \delta_{4}$ on calciumdependent inactivation of Cav1.4, and identified no differences in the inactivation kinetic between $\mathrm{Ba}^{2+}$ - and $\mathrm{Ca}^{2+}$-driven currents. The percentage of remaining current during 250-ms test pulse to 2 and $12 \mathrm{mV}$ (which was the voltage of the (lane 8).
$91 \%$ for $\mathrm{Ca}^{2+}$, with no significant difference observed $(P=$ $0.56)$. No difference was found over a voltage range from -40 to $+40 \mathrm{mV}$, as also previously noted with $\alpha_{2} \delta_{1}$ using the same experimental settings. ${ }^{11}$ Similar results were fairly recently reported also for human $\alpha_{2} \delta_{4}$ subunits. ${ }^{10}$

Since our primary aim was to test the feasibility of exon skipping as a potential therapeutic approach for $\alpha_{2} \delta_{4}$ mutant subunits, we assessed the functionality of proteins missing either three (E23-24-25) or four (E23-24-25-26) exons. Neither construct increased current densities and both were comparable to our negative control (Cav1.4, $\beta 3$, and pIRES vector) (Fig. 5D; Table 2). We also characterized the electrophysiological properties of the E25b isoform, which we expected to mimic the c.2451insC mutant, and the $\Delta \mathrm{E} 16$ variant: none of these variants evoked a change in current density levels (Figs. $5 \mathrm{~A}, 5 \mathrm{~B}, 5 \mathrm{D}$; Table 2), and activation gating properties of Cav1.4 were not altered with any of the variants for which we were able to obtain an activation curve (Fig. 5C; Table 2). We finally tested whether we could exert a possible dominant negative effect of the different splicing isoforms or the mutant by expressing them together with full-length $\alpha_{2} \delta_{4}$ in a 1:1 ratio. We ruled out a possible dominant negative effect of the different splicing isoforms as no changes in current densities were appreciated if compared to $\alpha_{2} \delta_{4}$ alone (Fig. 6).

\section{Discussion}

We discovered that the previously described c.2451insC mutation, ${ }^{7}$ causing retinopathy in mice, apart from truncating the ORF of the gene after the first Cache domain, also affects splicing. The mutation favors inclusion of a newly identified alternatively spliced exon, E25b, which also terminates the ORF. The mutation created new ESSs on E25 and promoted E25b inclusion in place of E25. In vivo, the mutation mainly affected E25, impairing its incorporation in mature mRNA. With use of a minigene reporter system in HEK293T cells, its effect is instead to favor E25b inclusion, without significantly affecting E25 levels. The observed difference of action could be due to the presence of different trans-acting splicing factors in the two analyzed systems: HEK293T cell line and mouse retinae. Differences in the impact of nonsense-mediated decay on the overall levels of transcripts ${ }^{32}$ might also be involved,

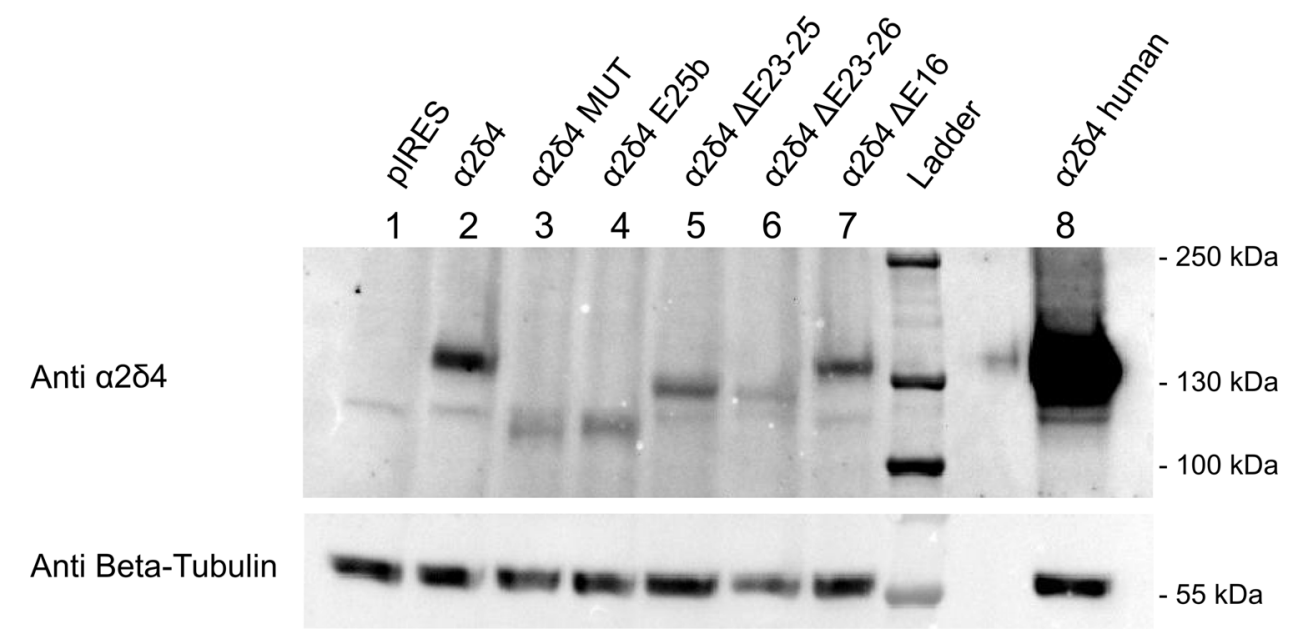

FIgURE 4. Expression of the different $\alpha_{2} \delta_{4}$ variants in tsA-201 cells after transfection. A specific band for $\alpha_{2} \delta_{4}$ is visible following tsA-201 transfection with Cav1.4 $\alpha_{1}, \beta_{3}$, and constructs encoding all the different $\alpha_{2} \delta_{4}$ variants utilized (lanes 2-7). The $\alpha_{2} \delta_{4}$ band changes in size accordingly to the relative length of the expressed variant. In the negative control (lane 1), $\alpha_{2} \delta_{4}$ is not detectable, proving the absence of endogenous $\alpha_{2} \delta_{4}$ in tsA-201 cells. A condition in which human $\alpha_{2} \delta_{4}$ was transfected $\left(\alpha_{2} \delta_{4}\right.$ human) has been added as an additional positive control 
A.
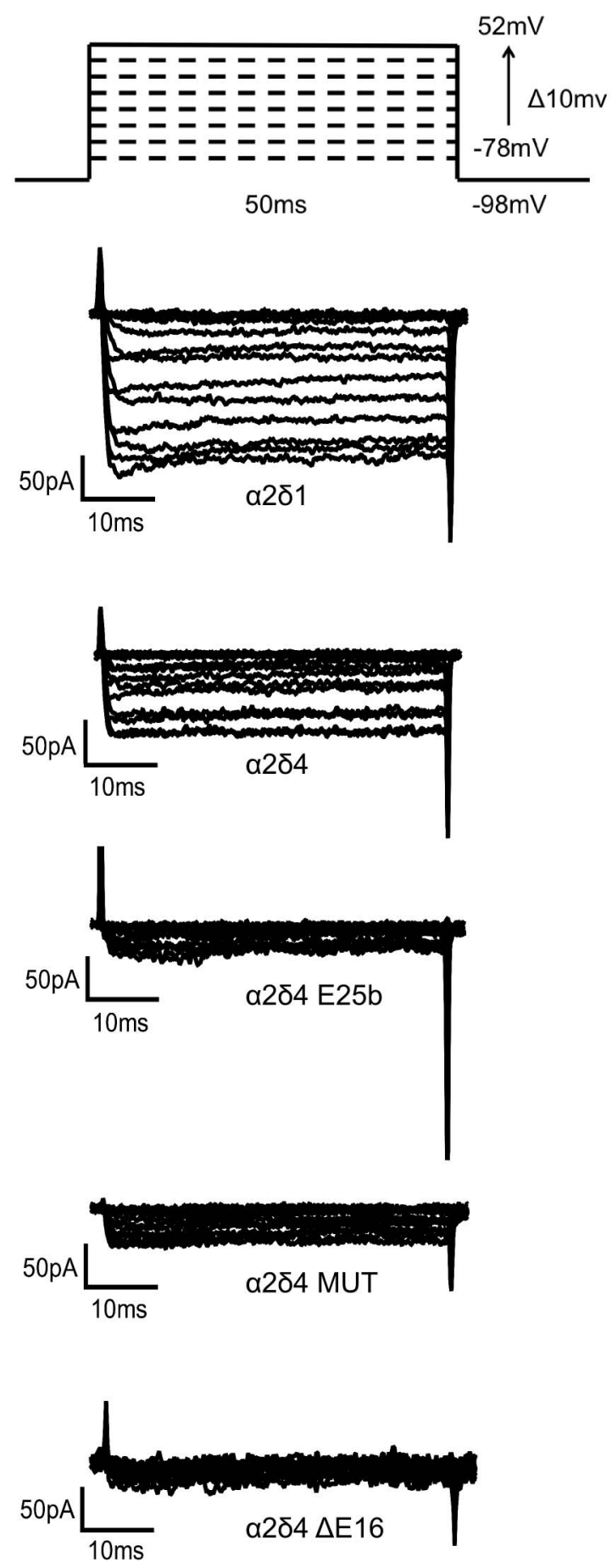

B.

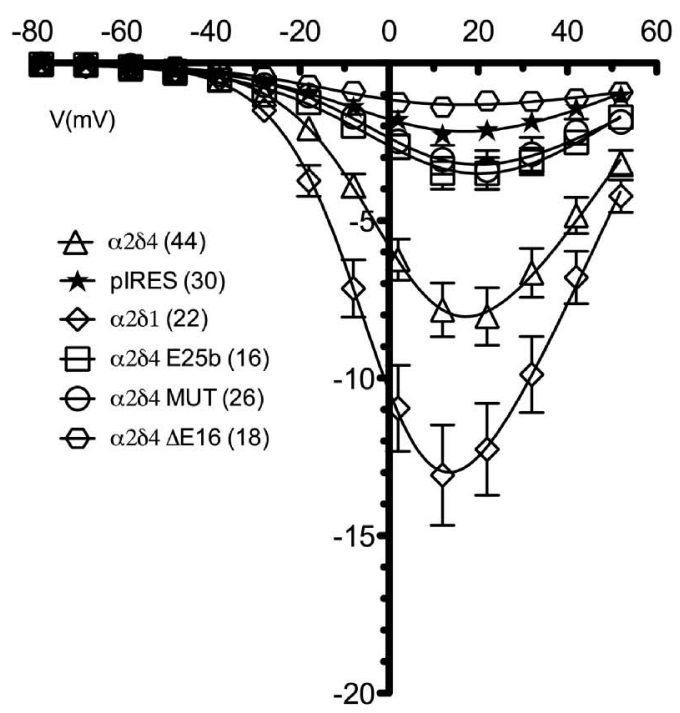

C.

Current Density $(\mathrm{pA} / \mathrm{pF})$

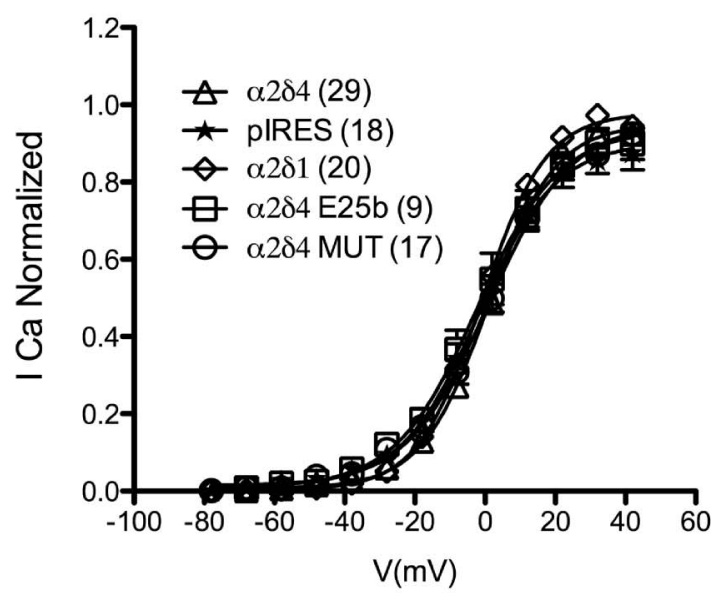

D.

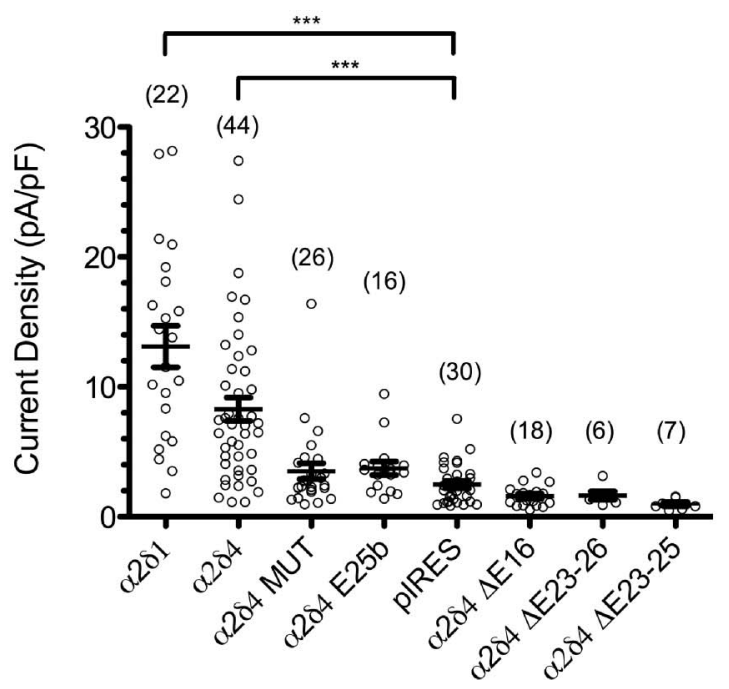

Figure 5. Effect of different $\alpha_{2} \delta_{4}$ variants on Cav1.4-mediated current density. (A) Scheme of the recording protocol and representative sample traces for some of the different tested conditions. (B) Representation of the changes in current density at the different voltages tested for $\alpha_{2} \delta_{1}, \alpha_{2} \delta_{4}$ $\alpha_{2} \delta_{4}$ E25b, $\alpha_{2} \delta_{4}$ MUT (c.2451insC), $\alpha_{2} \delta_{4} \Delta$ E16, pIRES (empty vector). Mean values, SEM, and the best fits of the current voltage relation are shown. The total number of cells considered for each curve is reported in parentheses. (C) Voltage-dependent activation curve of $\alpha_{2} \delta_{1}, \alpha_{2} \delta_{4}, \alpha_{2} \delta_{4} \mathrm{E} 25 \mathrm{~b}$, $\alpha_{2} \delta_{4}$ MUT (c.2451insC), $\alpha_{2} \delta_{4} \Delta$ E16, pIRES (empty vector). (D) Maximal current densities of cells measured in the presence of different $\alpha_{2} \delta_{4}$ subunits in comparison to $\alpha_{2} \delta_{1}$ and pIRES. For each condition, mean and SEM are shown. Selected pairs of conditions were compared using Kruskal-Wallis test followed by Dunn's multiple comparison posttest $\left({ }^{* * *} P<0.001\right)$ 
TABLE 2. Biophysical Parameters of Different $\alpha_{2} \delta$ Constructs Coexpressed With Cav1.4 $\alpha_{1}$ and $\beta_{3}$

\begin{tabular}{|c|c|c|c|c|c|}
\hline & $\mathrm{CD}_{\max }$ & $\mathbf{V}_{0.5}$, act & $\mathbf{K}_{\text {act }}$ & $\mathbf{V}_{0.5}$, inact & $\mathbf{K}_{\text {inact }}$ \\
\hline$\alpha 2 \delta 4$ & $8.28 \pm 0.90^{*} N=44$ & $1.64 \pm 0.55 N=29$ & $9.87 \pm 0.30 N=29$ & $-20.24 \pm 2.29 N=15$ & $-13.00 \pm 0.97 N=15$ \\
\hline$\alpha 2 \delta 4$ E25b & $3.74 \pm 0.52 N=16$ & $-0.98 \pm 2.61 N=9$ & $11.37 \pm 1.13 N=9$ & ND & ND \\
\hline$\alpha 2 \delta 4$ mut & $3.51 \pm 0.61 N=26$ & $1.03 \pm 1.76 \mathrm{~N}=17$ & $10.99 \pm 0.57 N=17$ & ND & ND \\
\hline$\alpha 2 \delta 1$ & $13.12 \pm 1.60^{*} N=22$ & $-1.05 \pm 0.44 N=20$ & $9.11 \pm 0.17 N=20$ & $-17.81 \pm 2.27 N=29$ & $-14.39 \pm 1.28 N=29$ \\
\hline pIRES & $2.49 \pm 0.29 N=30$ & $0.52 \pm 2.38 N=18$ & $10.80 \pm 0.69 N=18$ & ND & ND \\
\hline $\mathrm{A} 2 \delta 4 \Delta \mathrm{E} 16$ & $1.59 \pm 0.19 N=18$ & ND & ND & ND & ND \\
\hline A2 $\delta 4 \Delta \mathrm{E} 23-26$ & $1.64 \pm 0.33 N=6$ & ND & ND & ND & ND \\
\hline $\mathrm{A} 2 \delta 4 \Delta \mathrm{E} 23-25$ & $0.97 \pm 0.16 N=7$ & ND & ND & ND & ND \\
\hline
\end{tabular}

Experiments were conducted in tsA-201 cells using $15 \mathrm{mM} \mathrm{Ca}^{2+}$ as charge carrier. Data are given as mean \pm SEM. For multiple comparisons $\left(\mathrm{CD}_{\text {max }} ; \mathrm{V}_{0.5 \text { act }} ; \mathrm{K}_{\mathrm{act}}\right)$, significance is given in relation to Cav1.4 $\alpha 1+\beta_{3}+$ pIRES. For single comparison $\left(\mathrm{V}_{0.5}\right.$ inact $\left.; \mathrm{K}_{\text {inact }}\right)$, significance is given in relation to Cav1.4 $\alpha 1+\beta_{3}+\alpha_{2} \delta_{1}$. Data for $\alpha_{2} \delta_{1} \mathrm{~V}_{0.5}$ inact and $\mathrm{K}_{\text {inact }}$ were taken from Burtscher et al. ${ }^{11} \mathrm{CD}_{\text {max }}$, maximum current density; $\mathrm{V}_{0.5 \text {, act }}$, halfmaximum activation voltage; $K_{a c t}$, slope parameter of the activation curve; $V_{0.5}$, inact, half-maximum inactivation voltage; $K_{\text {inact }}$, slope parameter of the inactivation curve; $N$, sample size; ND, not determined.

$* P<0.001$, statistically significant differences.

especially in vivo where we see a general reduction of Cacna2d4 mRNA.

Our findings are also pathophysiologically relevant, since we confirmed the predicted role of murine $\alpha_{2} \delta_{4}$ as an accessory subunit of Cav1.4 by characterizing its biophysical properties. This subunit is able to increase current densities by 3 -fold in a heterologous expression system, in accordance with the changes in calcium influx previously described using human $\alpha_{2} \delta_{4}{ }^{4}$ Moreover, $\alpha_{2} \delta_{4}$ has no effect on the activation properties of Cav1.4 and is comparable to $\alpha_{2} \delta_{1}$ when inactivation is investigated. We therefore suggest that, since $\alpha_{2} \delta_{4}$ is highly expressed in the retina together with Cav1.4, where both localize to photoreceptor terminals,, $933-35$ this is likely the subunit helping trafficking of Cav1.4 to the plasma membrane of photoreceptors, as it is able to mediate the same effect on the channel in a heterologous system.

The $\alpha_{2} \delta_{4}$ isoform resulting from E25b inclusion completely lacks the $\delta$ peptide, important for membrane anchoring. ${ }^{6,36}$ Even if $\alpha_{2} \delta$ subunits lacking the membrane anchor site are still able to enhance calcium currents, they lose this ability when the whole $\delta$ peptide is deleted. ${ }^{36,37}$ Indeed, $\alpha_{2} \delta_{4}$ c. 2451 insC and $\alpha_{2} \delta_{4}$ E25b did not increase current densities. Therefore,

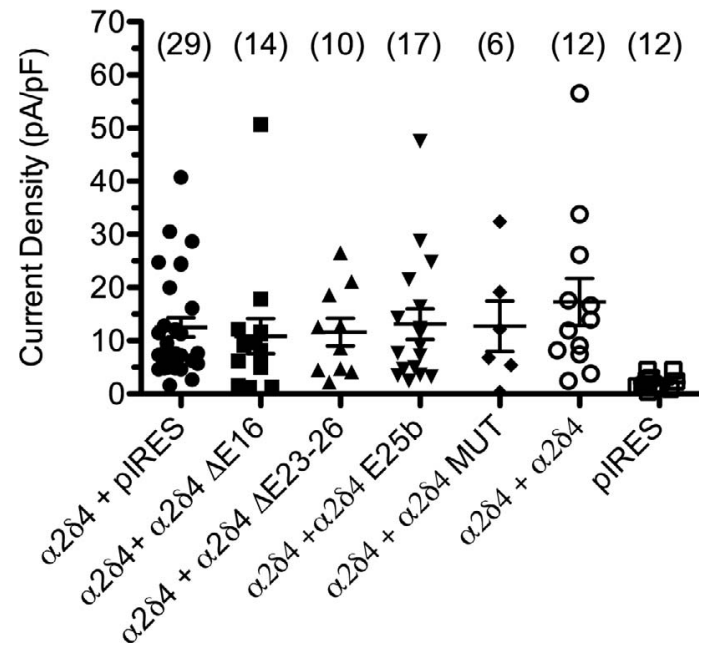

FiguRe 6. Effect of the coexpression of full-length $\alpha_{2} \delta_{4}$ with other $\alpha_{2} \delta_{4}$ variants. The full-length $\alpha_{2} \delta_{4}$ construct was cotransfected with equal amounts of other $\alpha_{2} \delta_{4}$ variants. No significant differences on maximal current density were observed in any of the tested conditions as compared with $\alpha_{2} \delta_{4}+\alpha_{2} \delta_{4}$, or with $\alpha_{2} \delta_{4}+$ pIRES. As a result no dominant negative effect of the different variants on $\alpha_{2} \delta_{4}$ activity was observable in the employed experimental conditions. the role of the $\alpha_{2} \delta_{4} \mathrm{E} 25 \mathrm{~b}$ splicing isoform in the retina remains elusive, as the protein would be secreted. Elucidation of the presence of a splicing event in human CACNA2D4 that resembles the effect of murine E25b incorporation would be important. Specifically, if an alternatively spliced exon truncating CACNA2D4 ORF between the two Cache domains exists also in the human gene, also in this case the c.2406C $\rightarrow \mathrm{A}$ mutation could favor this inclusion.

We show that the region encoded by exons 23 to 26 is of fundamental importance for the functionality of $\alpha_{2} \delta_{4}$. The removal of this portion indeed impairs the ability of $\alpha_{2} \delta_{4}$ to promote calcium currents mediated by Cav $1.4 \alpha_{1}$ subunits. The region encoded by these exons spans between the two Cache domains and is not part of any known domain of the protein. Moreover, in an $\alpha_{2} \delta_{1}$ paralogue, insertion of an HA tag in the corresponding region did not affect the protein's ability to mediate an increase in currents. ${ }^{36}$ This region may host glycosylation sites important for its functionality. ${ }^{37}$ This impairs our therapeutic strategy aimed at correcting the retinopathy caused by c. 2451 insC mutation, since this strategy was relying on the therapeutic skipping of exons 23 to 26 or 23 to 25 from transcripts in order to eliminate the frameshift mutation on E25 and restore the ORF downstream of it.

We described the presence of two newly identified splicing isoform (Cacna2d4 E25b and Cacna2d4 $\Delta \mathrm{E} 16$ ) that were unable to influence Cav1.4 currents. In keeping with previous studies, ${ }^{38}$ Cacna2d4 $\Delta \mathrm{E} 16$ is present at low levels in the analyzed CNS areas. Moreover, in a recent study, a smaller $(\sim 165 \mathrm{kDa}$ instead of $\sim 170 \mathrm{kDa}) \alpha_{2} \delta_{4}$ was detected by Western blots in mouse brain, possibly being $\alpha_{2} \delta_{4} \Delta \mathrm{E} 16 .{ }^{33}$ These results strengthen the current hypothesis ${ }^{39}$ that $\alpha_{2} \delta$ subunits may fulfill, in the retina and CNS, different functions not directly related to trafficking of calcium channels on the plasma membrane. The reason for occurrence of two different frameshift-causing mutations both on E25 in humans and mice and the presence of a splicing isoform that recapitulates the effect of the mutation in mice remains elusive. It is likely that the mutation, by mimicking the effect of E25b inclusion, offers a specific advantage in heterozygosity (overdominance) in the eye or in other districts where $\alpha_{2} \delta_{4}$ is normally expressed. This would allow the conservation of different mutations sharing the same mimicking effect.

The effect that mutations in the coding region of genes have on splicing is generally underestimated: It is today believed that more than a quarter of these mutations interfere with proper splicing. ${ }^{40-42}$ As we showed in this work, early investigation of how splicing patterns change in response to a mutation is of pivotal importance for the implementation of therapies involving antisense-mediated splicing-correcting approaches. 
To this end, splicing-reporter minigene assays can constitute a precious ally in this effort: We described here for the first time the design and use of a minigene system able to properly recall all splicing events across $6.5 \mathrm{~Kb}$ and six different exons. With the implementation of more efficient gene synthesis services this powerful approach could become relatively easy to use.

\section{Acknowledgments}

The authors thank Klaus Schicker, Dagmar Knoflach, Margherita Grasso, and Valerio del Vescovo for valuable experimental support. Supported by the Italian Ministry of Health Project GR-20081136933 (MAD) and Austrian Science Funds FWF-P26881-B23 (AK).

Disclosure: N. Bacchi, None; A. Messina, None; v. Burtscher, None; E. Dassi, None; G. Provenzano, None; Y. Bozzi, None; G.C. Demontis, None; A. Koschak, None; M.A. Denti, None; S. Casarosa, None

\section{References}

1. Bacchi N, Casarosa S, Denti MA. Splicing-correcting therapeutic approaches for retinal dystrophies: where endogenous gene regulation and specificity matter. Invest Ophthalmol Vis Sci. 2014;55:3285-3294.

2. Siva K, Covello G. Denti MA. Exon-skipping antisense oligonucleotides to correct missplicing in neurogenetic diseases. Nucleic Acid Ther. 2014;24:69-86.

3. Benchaouir R, Goyenvalle A. Splicing modulation mediated by small nuclear RNAs as therapeutic approaches for muscular dystrophies. Curr Gene Ther. 2012;12:179-191.

4. Qin N, Yagel S, Momplaisir M, Codd EE, Andrea MRD. Molecular cloning and characterization of the human voltagegated calcium channel $\alpha 2 \delta 4$ subunit. Mol Pharmacol. 2002; 62:485-496.

5. Dolphin AC. The $\alpha 2 \delta$ subunits of voltage-gated calcium channels. Biochim Biophys Acta. 2013;1828:1541-1549.

6. Davies A, Kadurin I, Alvarez-Laviada A, et al. The alpha2delta subunits of voltage-gated calcium channels form GPI-anchored proteins, a posttranslational modification essential for function. Proc Natl Acad Sci U S A. 2010;107:1654-1659.

7. Wycisk KA, Budde B, Feil S, et al. Structural and functional abnormalities of retinal ribbon synapses due to Cacna2d4 mutation. Invest Ophthalmol Vis Sci. 2006;47:3523-3530.

8. Wycisk KA, Zeitz C, Feil S, et al. Mutation in the auxiliary calcium-channel subunit CACNA2D4 causes autosomal recessive cone dystrophy. Am J Hum Genet. 2006;79:973-977.

9. Knoflach D, Kerov V, Sartori SB, et al. Cav1.4 IT mouse as model for vision impairment in human congenital stationary night blindness type 2. Channels. 2013;7:503-513.

10. Lee A, Wang S, Williams B, Hagen J, Scheetz TE, Haeseleer F. Characterization of Cav1.4 complexes $(\alpha 11.4, \beta 2, \alpha 2 \delta 4)$ in HEK293T cells and in the retina. J Biol Chem. 2014;290:15051521.

11. Burtscher V, Schicker K, Novikova E, et al. Spectrum of Cav1.4 dysfunction in congenital stationary night blindness type 2 . Biochim Biophys Acta. 2014;1838:2053-2065.

12. Ball SL, Powers PA, Shin H-S, Morgans CW, Peachey NS, Gregg RG. Role of the beta(2) subunit of voltage-dependent calcium channels in the retinal outer plexiform layer. Invest Ophthalmol Vis Sci. 2002;43:1595-1603.

13. Edgar R, Domrachev M, Lash AE. Gene Expression Omnibus: NCBI gene expression and hybridization array data repository. Nucleic Acids Res. 2002;30:207-210.

14. Bolger AM, Lohse M, Usadel B. Trimmomatic: a flexible trimmer for Illumina sequence data. Bioinformatics. 2014;30: 2114-2120.
15. Kim D, Pertea G, Trapnell C, Pimentel H, Kelley R, Salzberg SL. TopHat2: accurate alignment of transcriptomes in the presence of insertions, deletions and gene fusions. Genome Biol. 2013;14:R36.

16. Thorvaldsdóttir H, Robinson JT, Mesirov JP. Integrative Genomics Viewer (IGV): high-performance genomics data visualization and exploration. Brief Bioinform. 2013;14:178192.

17. Reese MG, Eeckman FH, Kulp D, Haussler D. Improved splice site detection in Genie. J Comput Biol. 1997;4:311-323.

18. Piva F, Giulietti M, Nocchi L, Principato G. SpliceAid: a database of experimental RNA target motifs bound by splicing proteins in humans. Bioinformatics. 2009;25:1211-1213.

19. Wang W, El-Deiry W. Large Insertions: Two Simple Steps Using QuikChange II Site-Directed Mutagenesis Kits. Agilent Technologies Technical Note. 2010.

20. Venkatachalan SP, Bushman JD, Mercado JL, Sancar F, Christopherson KR, Boileau AJ. Optimized expression vector for ion channel studies in Xenopus oocytes and mammalian cells using alfalfa mosaic virus. Pflugers Arch Eur J Physiol. 2007; $454: 155-163$.

21. Wong W, Newell EW, Jugloff DGM, Jones OT, Schlichter LC. Cell surface targeting and clustering interactions between heterologously expressed PSD-95 and the Shal voltage-gated potassium channel, Kv4.2. J Biol Chem. 2002;277:2042320430.

22. Sinnegger-Brauns MJ, Huber IG, Koschak A, et al. Expression and 1,4-dihydropyridine-binding properties of brain L-type calcium channel isoforms. Mol Pharmacol. 2009;75:407-414.

23. Castellano A, Wei X, Birnbaumer L, Perez-Reyes E. Cloning and expression of a third calcium channel beta subunit. $J$ Biol Chem. 1993;268:3450-3455.

24. Ellis SB, Williams ME, Ways NR, et al. Sequence and expression of mRNAs encoding the alpha 1 and alpha 2 subunits of a DHPsensitive calcium channel. Science. 1988;241:1661-1664.

25. Klugbauer N, Lacinova L, Hobom M, Hofmann F. Molecular diversity of the calcium channel $\alpha 2 \delta$ subunit. J Neurosci. 2000;19:684-691.

26. Dolphin AC, Wyatt CN, Richards J, et al. The effect of $\alpha 2-\delta$ and other accessory subunits on expression and properties of the calcium channel $\alpha 1$ G. J Physiol. 1999;519(pt 1):35-45.

27. Hobom M, Dai S, Marais E, et al. Neuronal distribution and functional characterization of the calcium channel a $2 \mathrm{~d}-2$ subunit. Eur J Neurosci. 2000;12:1217-1226.

28. Jones LP, Wei S, Yue DT. Mechanism of auxiliary subunit modulation of neuronal $\alpha 1 \mathrm{E}$ calcium channels. J Gen Physiol. 1998;112:125-143.

29. Felix R, Gurnett CA, De Waard M, Campbell KP, Roche IJ. Dissection of functional domains of the voltage-dependent Ca2+ channel $\alpha 2 \delta$ subunit. J Neurosci. 1997;17:6884-6891.

30. Sipos I, Pika-Hartlaub U, Hofmann F, Flucher BE, Melzer W. Effects of the dihydropyridine receptor subunits gamma and alpha2delta on the kinetics of heterologously expressed L-type Ca2+ channels. Pflugers Arch Eur J Physiol. 2000;439:691699.

31. Koschak A, Reimer D, Walter D, et al. Cav1.4alpha1 subunits can form slowly inactivating dihydropyridine-sensitive L-type $\mathrm{Ca} 2+$ channels lacking Ca2+-dependent inactivation. J Neurosci. 2003;23:6041-6049.

32. Nicholson P, Yepiskoposyan H, Metze S, Zamudio Orozco R, Kleinschmidt N, Mühlemann O. Nonsense-mediated mRNA decay in human cells: mechanistic insights, functions beyond quality control and the double-life of NMD factors. Cell Mol Life Sci. 2010;67:677-700.

33. De Sevilla Müller LP, Liu J, Solomon A, Rodriguez A, Brecha NC. Expression of voltage-gated calcium channel $\alpha(2) \delta(4)$ subunits in the mouse and rat retina. J Comp Neurol. 2013;521:24862501. 
34. Busquet P, Nguyen NK, Schmid E, et al. CaV1.3 L-type Ca2+ channels modulate depression-like behaviour in mice independent of deaf phenotype. Int J Neuropsychopharmacol. 2010;13:499-513.

35. Specht D, Wu S-B, Turner P, et al. Effects of presynaptic mutations on a postsynaptic Cacna1s calcium channel colocalized with mGluR6 at mouse photoreceptor ribbon synapses. Invest Ophthalmol Vis Sci. 2009;50:505-515.

36. Kadurin I, Alvarez-Laviada A, Ng SFJ, et al. Calcium currents are enhanced by $\alpha 2 \delta-1$ lacking its membrane anchor. $J$ Biol Chem. 2012;287:33554-33566.

37. Gurnett CA, De Waard M, Campbell KP. Dual function of the voltage-dependent $\mathrm{Ca} 2+$ channel alpha 2 delta subunit in current stimulation and subunit interaction. Neuron. 1996;16: 431-440.

38. Schlick B, Flucher BE, Obermair GJ. Voltage-activated calcium channel expression profiles in mouse brain and cultured hippocampal neurons. Neuroscience. 2012;167:786-798.

39. Dolphin AC. Calcium channel auxiliary $\alpha 2 \delta$ and $\beta$ subunits: trafficking and one step beyond. Nat Rev Neurosci. 2012;13: 542-555.
40. López-Bigas N, Audit B, Ouzounis C, Parra G, Guigó R. Are splicing mutations the most frequent cause of hereditary disease? FEBS Lett. 2005;579:1900-1903.

41. Sterne-Weiler T, Howard J, Mort M, Cooper DN, Sanford JR. Loss of exon identity is a common mechanism of human inherited disease. Genome Res. 2011;21:1563-1571.

42. Lim KH, Ferraris L, Filloux ME, Raphael BJ, Fairbrother WG. Using positional distribution to identify splicing elements and predict pre-mRNA processing defects in human genes. Proc Natl Acad Sci U S A. 2011;108:11093-11098.

43. Whittaker CA, Hynes RO. Distribution and evolution of von Willebrand/integrin A domains: widely dispersed domains with roles in cell adhesion and elsewhere. Mol Biol Cell. 2002; 13:3369-3387.

44. Springer TA. Complement and the multifaceted functions of VWA and integrin I domains. Structure. 2006;14:1611-1616.

45. Vivek A, Aravind L. Cache - a signaling domain common to channel subunits and a class of prokaryotic chemotaxis receptors. Trends Biochem Sci. 2000;25:535-537. 